\title{
The Research of Smart Ex-telemetry System of Launch Vechilce
}

\author{
Feng Tieshan ${ }^{1}$, Li Daquan ${ }^{1}$, Lan Kun ${ }^{1}$ \\ 1 Beijing institute of Astronautical Systems Engineering, Beijing, Fengtai Strict Beijing China, \\ fengtieshan1987@gmail.com \\ baiyou1987@163.com
}

\begin{abstract}
The article put forward a new idea to reduce the preparing time of launch vehicle. With the use of IPbased technology and artificial intelligence, every instrument in the launch vehicle can be connected with the high-speed and high-reliable data bus. Besides, the testing instruments on the launch pad will use the same protocol between the electronic instrument on board and off the board. In this way, a more simple testing process will be proposed which will reduce the preparing time and cut the cost of launching task. Many innovative details will be discussed in this paper. In Section I, practical issues for low-cost launching will be discussed in brief. In Section II, a general application scheme will be put forward to simplify the process of electronic design. Section III describes more details about the key technology to reduce the preparing time and launch cost. Section IV contains the models, results and analysis of the protocol simulation
\end{abstract}

Key words: launch cost, preparing time, IP-Based technology, Telemetry system, Simulation.

\section{Introduction}

The technology of launch vehicle stands for a country's ability of entering into the space autonomously. Since 1957, the first satellite is launched by the Soviet Union, the humans are pushing forward the technology of launching vehicle continuously. More than 500 lauches have been made to explore the outer space. In recent several modern locality conflicts, the military affairs have been revealed more and more important. At the same time, the weakness of the traditional space telemetry, test and control also restrict the application of launch vehicle. Besides when the natural disasters happens, the base stations of mobile phone are destroyed which makes troubles for rescuing. To sum up, it is important that reducing the launching time means a lot for a launch vehicle. For the war happens in future, because of the weakness of communication system of space-based, it is also important to enhancing the ability of rapidly-launching. Considering what is mentioned above, it is not hard to come to a conclusion that dropping down the cost, promoting the smart-test ability , realizing the low-cost and high-speed test is of significance for launch vehicles especially for the commercial launch vehicles.

\section{Common architecture}

The implement of smat-testing launching pad relies on the advanced electronic topology architecture and high-reliability transferring protocol. For this time, in the international aerospace launching application, ESA and NASA both take the TTE as the next generation high-speed data bus. This protocol is implemented based on AS6802 timing synchronization protocol, which is of great competing superiority. In conclude, the ability of networking and intelligentizing for launch vehicle has been the main developing stream. As while, the progress of networking technology also provide a new moment to implement the smart test of launching measuring system.

Different from the traditional measuring methods, in order to implement the fast testing system, the transferring protocol for the ground test and on-board test should be unified by one same protocol, which will increase the launching efficiency. Besides, the telemetry system also needs the ability of high reliability and expandable. However, the traditional networking transferring protocol and Timetrigged Ethernet can not provide the commandrespond mechanism. As a result, even the 
message can be transferred to the telemetry net through two different ways, we can not decide whether the destination node has received the message correctly. So we put forward a new application, which will in use of the physical layer of Ethernet, while the other communication layers will referring to 1553B protocol. The topology of system can be seen in fig-1.

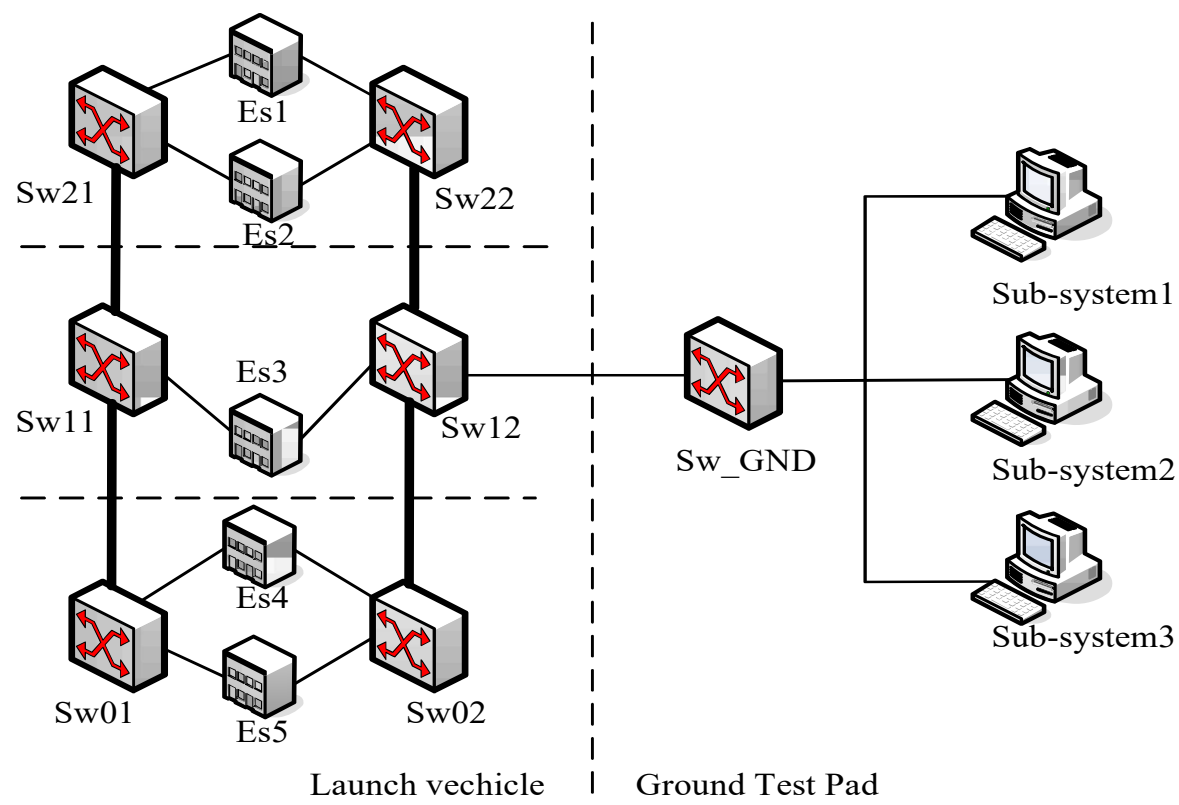

Fig 1 Testing Topology of Telemetry System

In Fig1, there are two parts for the testing topology of telemetry system. The first part is launch vehicle part, the other part is ground test pad. In launch vehicle part, there are two different electronic circult board. For the first one is switch electronic board, which mainly swap the transferring message from the input port to the correct destination port according to the configuration table in local equipment. For the second one, every equipment on the network should set the appointed electronic board to achieve the network-communication function. The second part is Ground Test Pad. In this part, there are two kinds of equipment, the switch node and sub-system testing computer. The switch is used to send every message to specific node. While all the stations of different sub systems would be linked to the central switch node.

Traditionally, the telemetry community has used a time division method of packaging the data into link-level transmission data frames for transport over radio links. With the growth of the Internet, the transmission of the telemetry packets often looks like a computer to computer communications as more telecommunications are used for data acquisition and distribution. Data communication will occur over wireless and wired links as well as fiber optic links. The IP-Based Telemetry Network is optimized from the traditional Ethernet technology. The equipments in the system will be distributed with an unique IP address. Besides all the network equipments will also have a MAC address when they were manufactured. Depending on the real-time protocol (like IEEE 1588), the whole system will have a relatively accurate operational clock.

The IPTN communication profile is shown in Fig. 2.

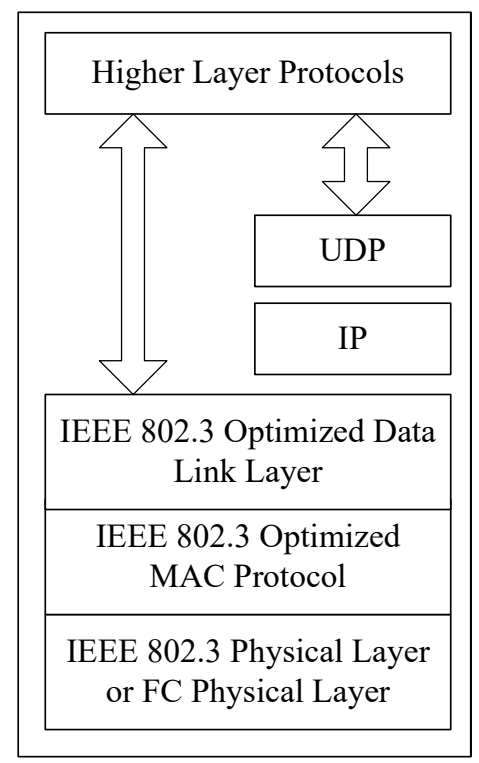

Fig.2. IPTN communication profile 
In Fig 2, IEEE 802.3 Physical Layer has been used for references. In order to reduce the weight of electric cable and the influence of electronic interference.The fiber (References as FCO) is also taken into account. The Media Access Control Layer and the Data Link Layer are the most important Layers which determine many integrated performance such as error rate ,packet delay and so on.With regard to the IEEE 802.3 Protocol Data Unit, IPTN comprises three additional fields which have been allocated on the first five bytes of the 'data' field of the IEEE 802.3 original frame. In this way, the system could match the specific field to recognise the correct frame and discard the redundant frame.What's more, as we do not destroy the original format of the IEEE 802.3 frame, the frames communicating in the network can also be transmitted in the industrial switchboard, which could unify the communicational protocols on board and ground. The highly mature UDP/IP services is invisible as well. The Internet Protocol sends or receives the network data of the net points. The IP is responsible for the fragmentation and reassembly of blocks of messages. This is required when the amount of data needed to sent is greater than the maximum IP data payload of a single frame. The User Datagram Protocol (UDP) parses data from one or multiple applications to the lower network protocols. However the Transmit Control Protocol is most used in the Ethernet, the timing of the receipt is non-deterministic. As a result, UDP is preferable in the IPTN. The frame format is shown in Fig 3.

\begin{tabular}{|c|c|c|c|c|c|c|c|}
\hline Preamble & $\begin{array}{c}\text { Start of frame } \\
\text { delimiter }\end{array}$ & $\begin{array}{c}\text { MAC } \\
\text { Destination }\end{array}$ & $\begin{array}{c}\text { MAC } \\
\text { Source }\end{array}$ & Tag & Length & Payload & CRC \\
\hline 7 octets & 1 octet & 6 octets & 6 octets & 4 & 2 octets & $46 ~ 1500$ octets & 4 octets \\
\hline
\end{tabular}
Fig.3. IPTN Frame Format

\section{Timing synchronization}

The disadvantage of Ethernet, opposite to an astronautical application, is a non intrinsic determinism of its access method to the physical support. To improve the bandwidth and real-time speciality, people put forward many ideas to make the telemetry system a determinate one. In spite of increasing the bandwidth to avoid conflicts, many other ways have been raised to confirm the real-time speciality of the whole system.

IPTN, as a flexible and reliable system, has many ways to improve its electronic features. First, using the IEEE 802.3 PHY Layer to guarantee its $100 / 1000 \mathrm{Mbps}$ Ethernet communicating ports.Secondly, comprises three additional fields which have been allocated on the first five bytes of the 'data' field of the IEEE 802.3 original frame.These new data fields are used to manage the redundant frames and to distinguish different data types.

The transmission of the telemetry data from the payload segment to the user segment and the commands from the user to the payload are typical communications problems encountered in many settings. Synchronization is a key technology for telemetry system design. Based on the IEEE802.3 original frame, the synchronization information is also included in the improved packet structure. While the net is under operation, the network equipment will control the system clock and finish the synchronization of the crystal oscillators. The network message could be divided into two types.

1) Event message: a message with time stamp.When the message is delilvering in the network, the network equipment could calculate the point-to-point delay with the help of time information stored in the event message.

2) General message: Opposed to event message, the general message does not contain any time information, this kind of message is mainly used to build the master-slave relationships.

Three steps are followed to achieve the synchronization of the whole net.

1) Establishing the master clock. In order to supervise the different clocks of all the network equipments, there should be a precise system clock in the whole system. This precise system clock could be obtained from Beidou II or GPS. Besides it can also be acquired by the high-precision clock source.

2) Synchronize the frequency of different clocks. The master clock send the sync message to the slave one, in which message there is time information stamped the sending time. The slave point receives this message and record the receiving time. While the same slave point receives at least two messages continuously, according to the sending intervals between contiguous messages. The slave point could adjust its clock frequency to the master point. In this way, all the equipments in the network could work under the unitive frequency. The regulation of clock frequency shows in fig 4.

Synchronize the time of different clocks. The event messages are used in the process of clock synchronization. The detailed steps are as follows. 


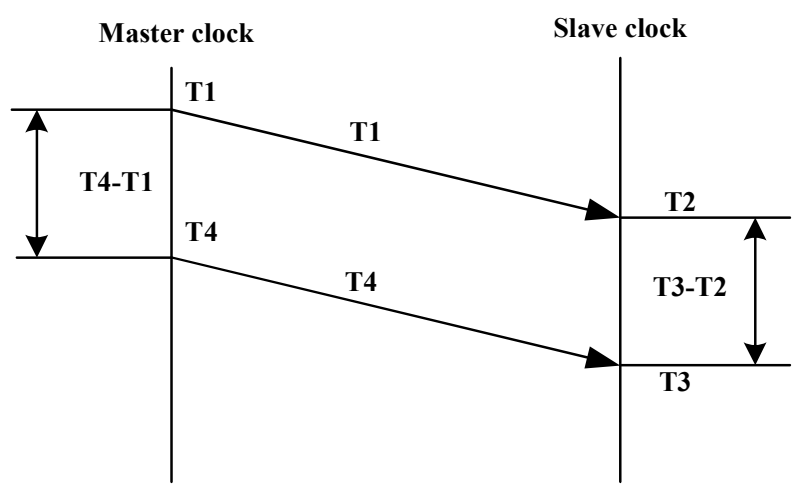

Fig.4. the Schematic diagram of frequency adjustment

1) The master clock send the sync message to the slave point, including the sending time T1;

2) The slave point records the receiving time T2;

3) The slave point returns the request messages to the master clock, and records the local sending time T3;

4) The master clock records the receiving time of request message T4. Then sending back the delay request message including the time T4 to the slave point;

5) The clock synchronization shows in the figure 5 .

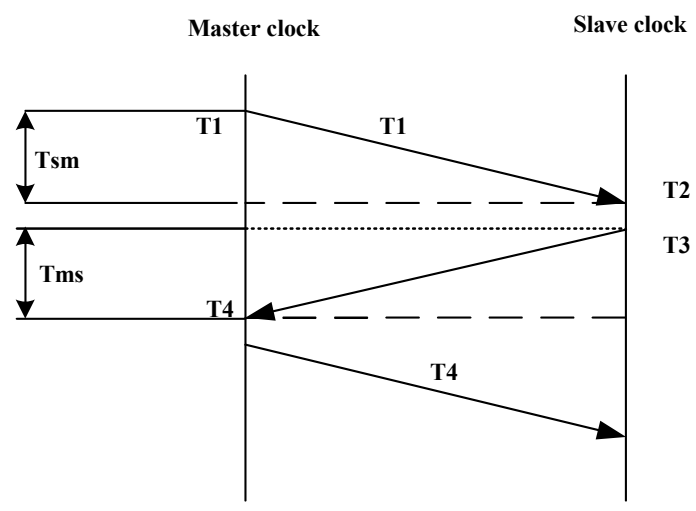

Fig.5. the Schematic diagram of timing adjustment

According to the four steps above, the slave clock could calculate the delay and off-time as follows.

$$
\begin{aligned}
& \text { Delay }=[(\mathrm{T} 4-\mathrm{T} 1)-(\mathrm{T} 3-\mathrm{T} 2)] / 2 \\
& \text { Offtime }=[(\mathrm{T} 2-\mathrm{T} 1)-(\mathrm{T} 4-\mathrm{T} 3)] / 2
\end{aligned}
$$

Depending on the route delay and off-time, the slave clock adjusts its clock to the master clock.
Data integration is a key function for telemetry syetem of launch vehicle. Compared with the traditional methods, the technology of net-based integration has its advantages in terms of reliability, data capacity, system intelligence, general duty and so on. In the launch vehicle data integration system, the configuration of the whole net is kernel, which decides some important designing targets, such as throughout capacity, packet loss probability and network delay. Considering the practical demanding, this article focuses on the configuration project of launch vehicle data integration system.

The appearance of virtual local area network tremendously promotes the development of real-time Ethernet. When the IPTN is working, there would be many virtual links flow into one equipment. In that case, this equipment must deal with these flows according to certain algorithm, which decides the performance of the whole network. In IPTN, the equipment implement a scheduler to manage the flows. The algorithm is mainly based on the Quality of Service (QoS). The scheduler would record the waiting time of every virtual links. According to the weight of links' priority, waiting time and the message length, the scheduler would send the proper link and update the weight of every items. The simulation will show the results of different scheduled algorithm.

\section{The algorithm of scheduling}

The algorithm of network frame scheduling has many mature methods, which is not available for launch vehicle. It is necessary to study the scheduling algorithm. Because the topology of network and end system are pre-settled, which results in a static network. Thanks to this special character, it is much easier for engineers to design the whole communication process of network. There are two different situations.

\section{Directly-distributed schedule}

The most important frame (we call it TimeStrict Frame,TSF) which needs to be transmitted at the very moment is scheduled as followed in Fig 6.

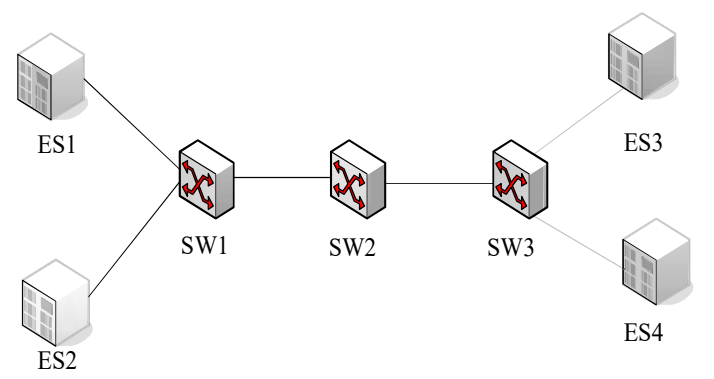

Fig.6. Directly-distributed schedule diagram 
Sw1, Sw2 and Sw3 are three switches in the network. When these switches transmit frames, they only work as transparent equipments, which means that when the valid data are being transmitted through the network by ES (End System), the TSF would be discarded or conveyed transparently. In that case, time-strict frame would be transmitted at the very moment. For instance, when ES1 is designed to transmit the frames to ES3, ES1 would send the frame the SW1. SW1 receives this frame and swap this frame directly to SW2. SW2 and SW3 would do the same thing to transmit this frame coutinuously.

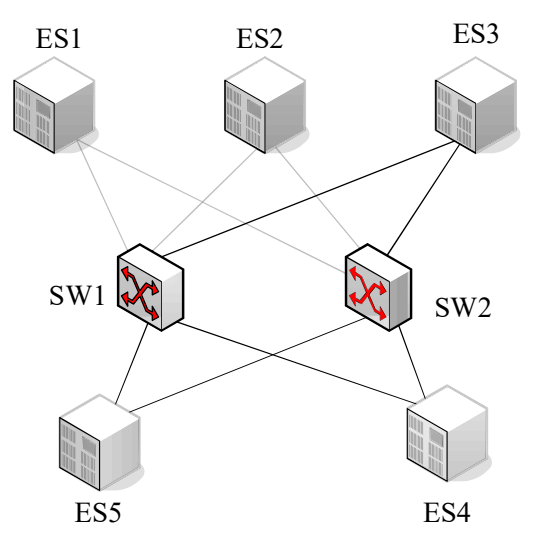

Fig.7. flowing frame in redundancy network
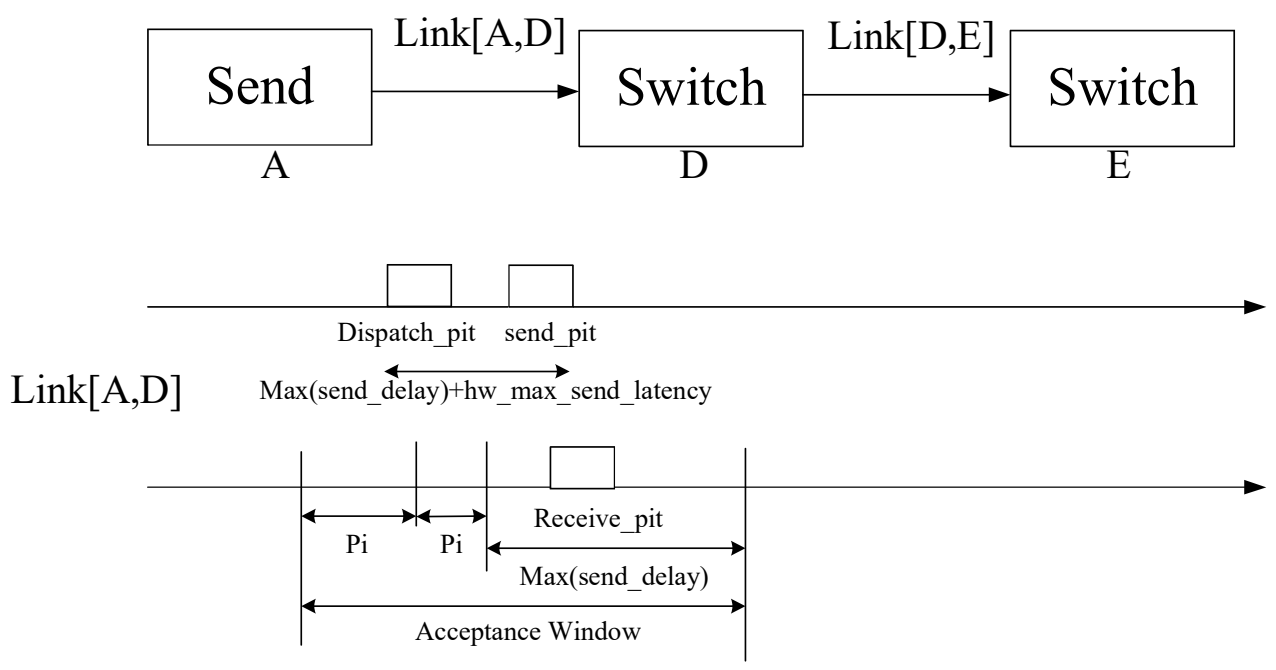

Fig. 8 .Directly-distributed schedule diagram

Fig 7 shows a redundancy network. In this situation, when the ports of SW in the network is busy, the TS Frame would not be discarded. On the other hand, SW would store this frame, and puth them into the fifo, waiting for the free time to swap this frame again. The destination node would receive two frames from the source node and according to the specific judging rules, the destination node would discard one frame and receive the other. What's more, when one frame is destroyed or lost in the net, the destination node would receive at least one frame.

\section{Store-distributed schedule}

In this part, we will directly get the algorithm. Firstly, we will define some definitions.

Dispatch_pit: the transferring time of the timestrict frame. This time is generated offline to trigger the dispatch of this kind of frame.

Send_pit: this moment refers to the transferring time of the first bit after the preamble.
Send_pit refers to the the scheduled moment at which time, just a new frame is being sent , resulting in postpone in net communication. Max(send_delay) stands for the maximum delay time between two different equipments. While hw_max_send_latency represents the settling time the circuit costs. So we can get:

$$
\begin{aligned}
\text { send_pit } \in & {[\text { dispatch_pit,dispatch _pit }} \\
& +\max (\text { send_delay }) \\
& \left.+h w_{-} \max _{-} \text {send_latency }\right]
\end{aligned}
$$

Receive_pit:

The receive_pit time represents the time the specific frame is being received.

$$
\text { receive_pit }=\text { send_pit }+ \text { link_latency }
$$

\section{the models of the net equipment}

The network equipment models are simulated with OPNET Modeler.There are two kinds of 
models in the simulation, communicating point and switching point.

The communicating point in the network is shown in figure 9.

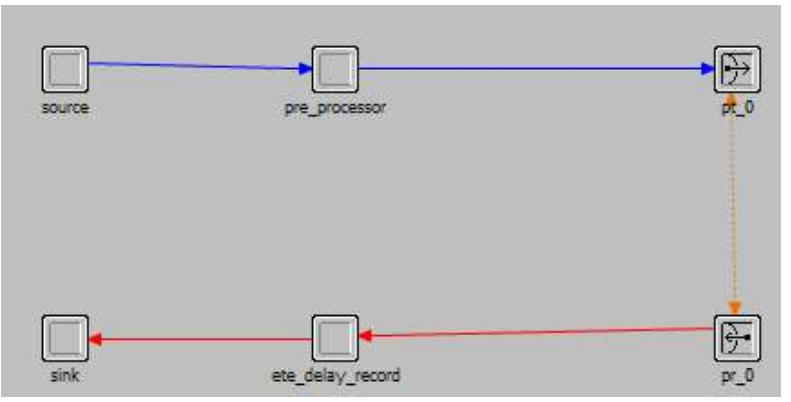

Fig.9. the model of communicating point

This node includes 6 parts. "Source" is used to generate the original data flow. "Pre-processor" is used to mark the timing stamp and assign the attributes for each virtual links. "pt_0" and "pr_0" is a mature function block to send and receive the network data. The results and synchronization is recorded by "Ete-delayrecord". At last, the data communicated in the network would flow into the "sink". The sink extract the useful information and destroy the packet.

The switching point receives the data flows from other communicating points. The schematic diagram of switch point is shown in figure 10 .

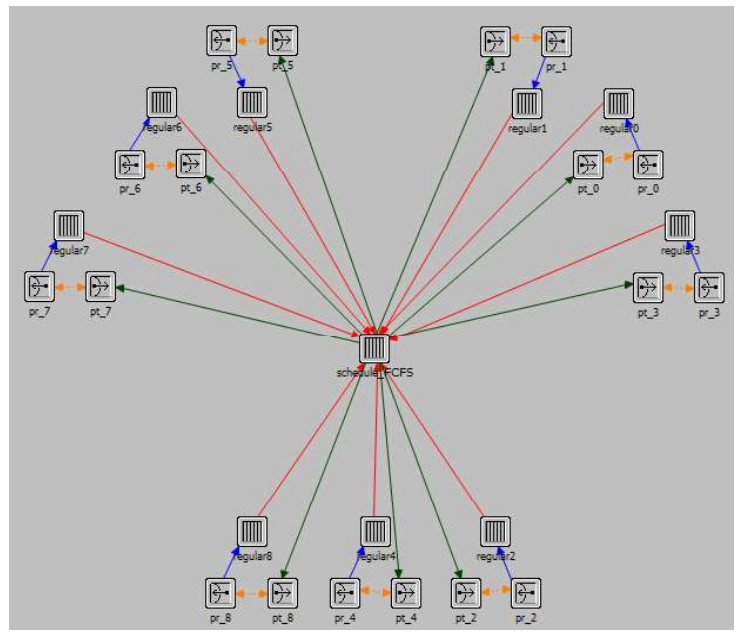

Fig. 10. the model of switching point

Every switching point has 9 ports to communicate with other points. There are 4 sub-models in each switching node. The function of "pr" and "pt" is used to receive and send the network packet. "Regular" is used to realize the MAC protocol of the switching point. "Scheduler" is the most important sub-model in the switching node. This block will pick up the certain attributes of every data flows and send it out according to some certain algorithm, which has been introduced briefly.

\section{2 the test scenario}

In this section, we consider a work-conserving system shared by multiple input processes under different service disciplines. Within each source, FIFO is assumed. In order to compare different schedule algorithms, two scenarios are built to prove the correctness of the whole system.

\subsubsection{Scenario I}

In scenario I, eight communicating nodes( node1 node8), acted as data sources send network frames to node9 by routing switch node in the center.

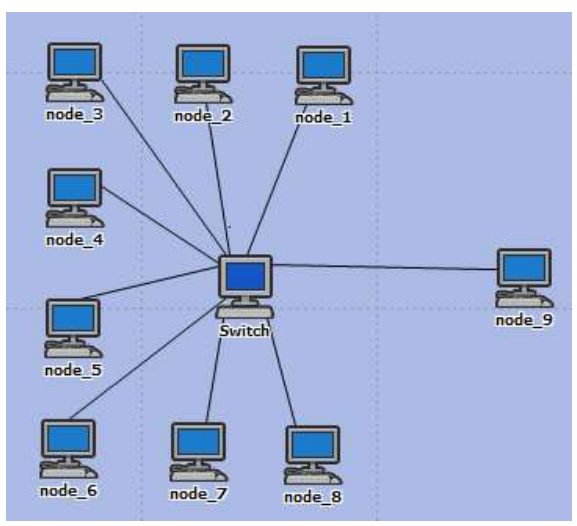

Fig.11. the topology of scenario I

In switch node, two different methods are used to testify the effects. One method is that every node in the network adopts the FIFO service discipline. The other one is that nodes in the network have strict priority. Assume that, if $i<j$, then the source I has a higher priority than the source j, which means that source $\mathrm{j}$ will not be served if there exists a workload from source I waiting for serving. Within the same source, FIFO is adopted. The results of the simulation would be shown in Fig 11.

\subsubsection{Scenario II}

In scenario II, an entire network would be built to check the correctness of the protocol. The topology of scenario II is showed in Fig12. 


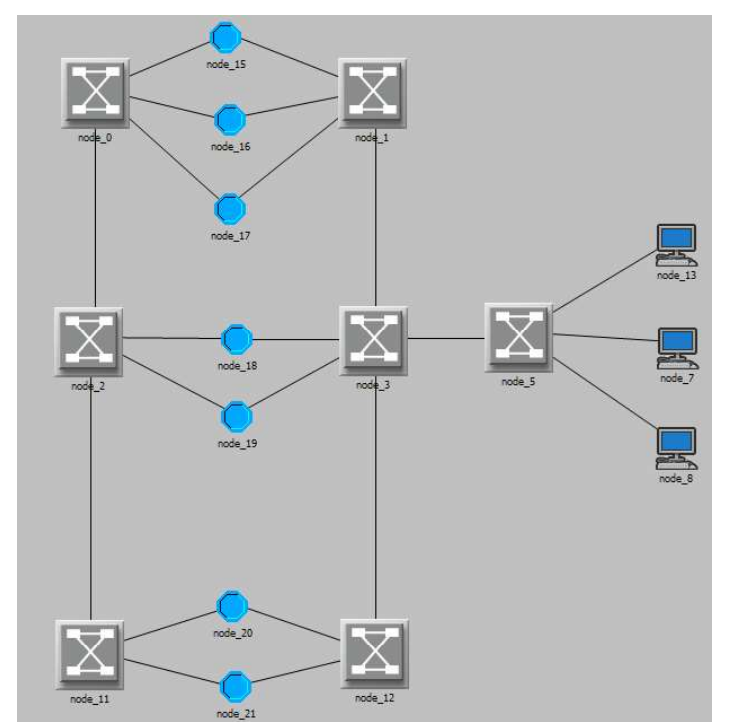

Fig.12. the topology of scenario II

In scenario II, the regular pattern of network nodes are listed in Table 1.

Table 1 Regular Pattern of Network Nodes

\begin{tabular}{l|l|l}
\hline Identifier & Flows Type & Mean Value \\
\hline Source1 & Exponential & 0.08 \\
Source2 & Exponential & 0.08 \\
Source3 & Exponential & 0.01 \\
Source4 & Exponential & 0.01 \\
Source5 & Constant & 0.08 \\
Source6 & Constant & 0.08 \\
Source7 & Constant & 0.08 \\
Source8 & Constant & 0.08 \\
Source9 & Constant & 0.01 \\
Source10 & Constant & 0.01 \\
Source11 & Constant & 0.01 \\
Source12 & Constant & 0.01 \\
Source13 & Constant & 0.01 \\
\hline
\end{tabular}

\section{Comparative Analysis of the two Scenarios}

In the simulation, the flow admission control relies on the algorithms for scheduling. In scenario I, two approaches are applied to the same scenario. The results of three nodes are picked. The results are depicted in figure 13 and figure 15.

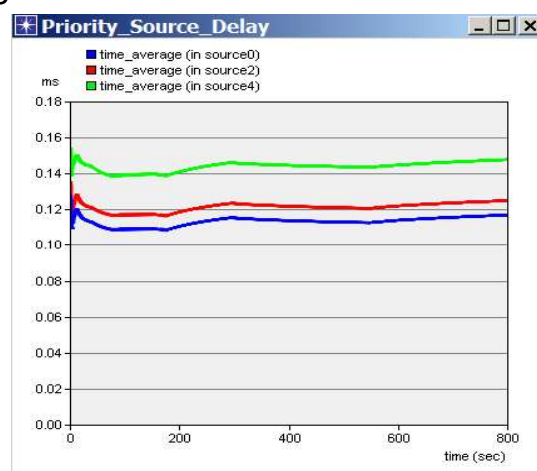

Fig.13. the results of FIFO schedule

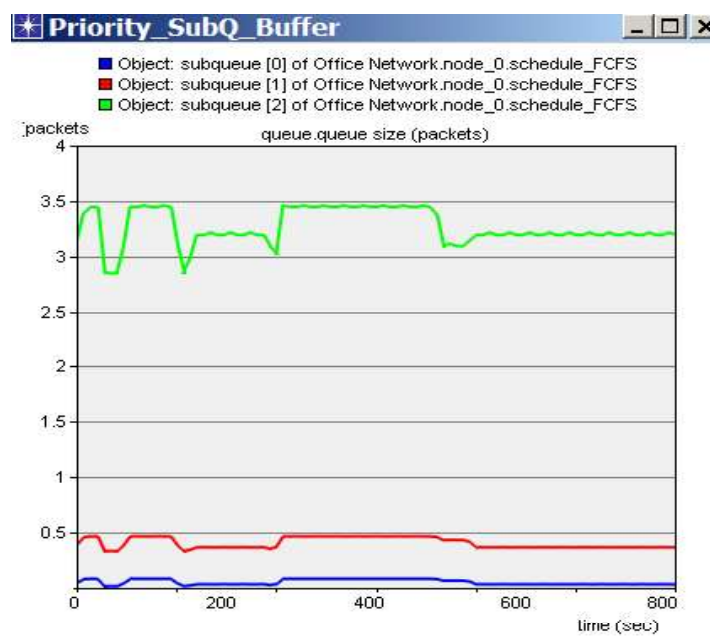

Fig.14 the results of strict priority schedule

The correctness of the IPTN protocol is proved in scenario II. The result is showed in figure 15.

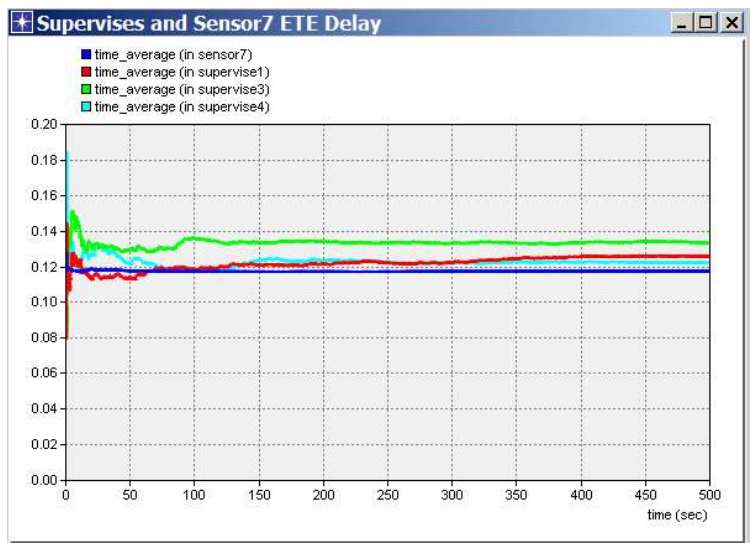

Fig.15. the topology of scenario I

The impact of the scheduling algorithm influences the real-time performance and the communicating process of the whole network. On these two scenarios, the trajectory approach shows two different trends, which implies the character of real-time. The full protocol stack is also proved in this simulation.

\section{Conclusion}

In order to reduce the preparing time for launching vehicle and decreasing the cost, the article put forward a new method to synthesize the on-board electronic system and on-ground test system. The traditional network communicating protocol can not meet the needs of real-time and high-reliability. Even some new real-time Ethernet protocol have settled these problems, the command frames transferring in the net can not be responded. The paper put forward a new method to make the equipments in the net communicate as 1553B-Based protocol. Besides, the equipments on the ground and the equipments on board can also be linked together. In this way, the interface between launch vehilce and ground can be simplified. 


\section{References}

[1] International Reference Guide to Space Launch System, Steven J.Isakowity,AIAA;1991

[2] Van Allen Richard E, The future of Responsive Space [J]. A Summary of the results and papers from the 5th Responsive Space Conference,2007

[3] Ed Allen, William U. Integrated Ground Operations Demonstration Units Testing Plans and Status [R]. AIAA-2012-5247,2012

[4] Jain, R. and Routhier, S.A "Packet Trains: measurements and a new model for computer network traffic". IEEE Journal on Selected Areas in Communications, 1986. 4: 986-995

[5] Randy Black, Mitch Fletcher."Next Generation Space Avionics: A Highly Reliable Layered System Implementation", IEEE Communications Magazine. 2000, 38 (4)2004 